\title{
Controlling the Nucleation of Metal Nanoislands on Two-dimensional Materials via Focused Ion Beam Patterning
}

Vera Zarubin ${ }^{1}$, Kate Reidy ${ }^{1}$, Yang $\mathrm{Yu}^{2}$, Ilya Charaev ${ }^{3}$, Joachim Dahl Thomsen ${ }^{1}$, Julian Klein ${ }^{4}$ and Frances Ross 4

${ }^{1}$ Massachusetts Institute of Technology (MIT), United States, ${ }^{2}$ Raith America, Inc., United States, ${ }^{3}$ Massachusetts Institute of Technology, United States, ${ }^{4}$ MIT, United States

The controlled creation of defects has been demonstrated to be a successful technique for modifying the intrinsic properties of two-dimensional materials (2DMs). In particular, patterning point defects, nanopores, and nanoribbons can enhance the electronic, optical, magnetic, and catalytic functionalities of 2DMs. ${ }^{1-3}$ At the same time, theoretical studies predict that metal adatoms and small clusters nucleate on point defects in $2 \mathrm{DMs}^{4-7}$ and that metal films grow in nanopores in graphene. ${ }^{8,9}$ This suggests that defect patterning may be extended to facilitate the self-assembly of metal nanoislands on 2DMs, enabling applications in plasmonics ${ }^{10}$ and single photon emission. ${ }^{11,12}$

Focused ion beams (FIBs) are well-suited for patterning 2DMs with nanometer precision milling and sputtering. By tuning irradiation parameters that influence the ion dose and beam profile, FIB patterning can be used for the controlled creation of point defects ${ }^{13}$ and sub-10nm features in suspended 2DMs. ${ }^{14-16}$ For the applications that require control of the locations of metal islands, it is important to optimize the irradiation parameters for metal nucleation. This will require an experimental understanding of defect creation mechanisms, nucleation kinetics, and metal-2DM interactions.

In this work, we study the structural changes that arise from FIB patterning of 2DMs and the effects of patterning on metal nucleation and growth (Fig. 1). We mechanically exfoliate bulk crystals to obtain layered 2DMs (graphene, $\mathrm{MoS}_{2}$, and $\mathrm{WSe}_{2}$ ). These are transferred to $\mathrm{SiN}$ transmission electron microscope (TEM) grids and annealed in ultra-high vacuum (UHV) to remove surface adsorbates, resulting in near atomically clean suspended membranes. Ions are delivered as arrays of spots and lines, either using $\mathrm{He}^{+}$in the Carl Zeiss ORION helium ion microscope (HIM) or using non-Ga ion species in the Raith VELION focused ion beam-scanning electron microscope (FIB-SEM). We calibrate the irradiation parameters to achieve accurate patterning with minimal damage to the 2DM membrane, and the patterned features are characterized in the Themis Z G3 scanning transmission electron microscope (STEM) (Fig. 2a).

Using these patterned 2DMs, we study the extent to which the defects, ion species, dose rate, and sample thickness affect the nucleation and growth of metals. Before depositing the metal it is important to clean the sample by annealing the 2DMs in UHV. The metal is then deposited via thermal evaporation without breaking vacuum. Fig. 2 shows representative results after the deposition of Au. At high deposition amounts, Au forms small islands around graphene nanopores (Fig. 2b), indicative of defect-mediated nucleation, corroborated by our pre-deposition STEM studies that reveal amorphized regions around each nanopore (Fig. 2a). Compared to graphene, $\mathrm{MoS}_{2}$ and $\mathrm{WSe}_{2}$ appear to exhibit fewer nucleation sites around patterned nanopores, possibly due to having less reactive defects. ${ }^{14}$ Depending on the spacing of the array, islands can attain their equilibrium shapes outside the amorphized regions. In particular, the islands are smaller for higher doses and tend towards equilibrium or larger sizes for lower doses. This can 
be used to automatically vary the size of Au islands. We study the evolution of the island shape and size during in situ TEM post-deposition annealing.

The templating and nucleation control strategy presented here can be generalized to anchoring other materials on 2DMs, such as Si and Ge via chemical vapor deposition or other metals via thermal and ebeam evaporation. This opens routes towards the directed self-assembly of semiconducting and metallic nanoislands on 2DMs with optimized charge transfer and strong light-matter interactions.

a)

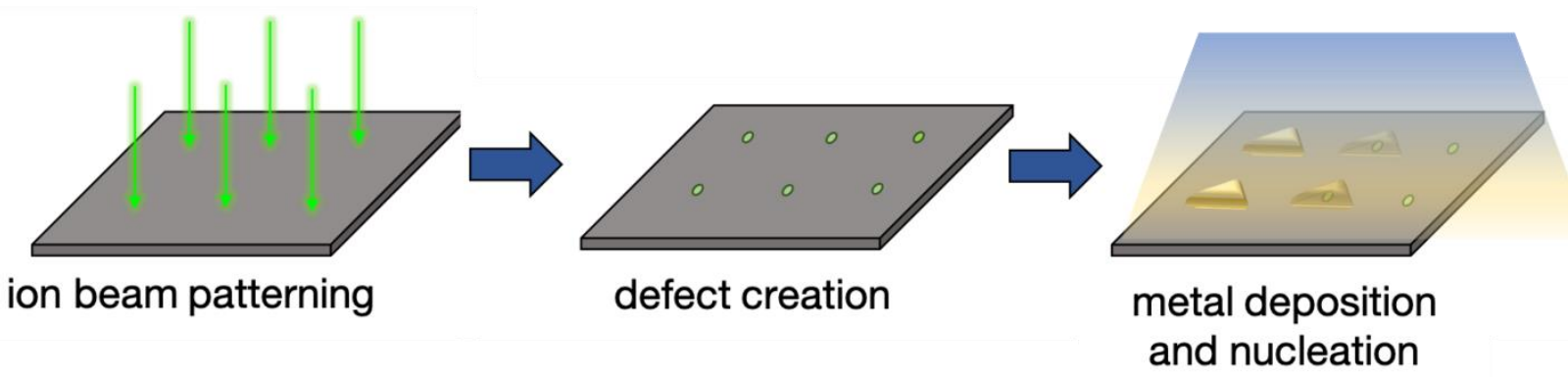

b)
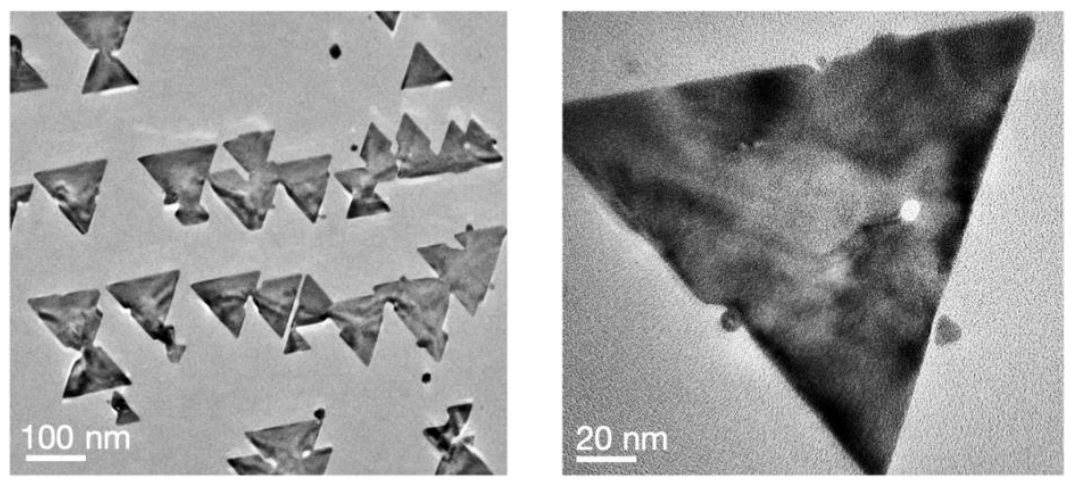

Figure 1. Figure 1: Overview of FIB patterning approach. a) Schematic of ion-beam tailored nucleation concept on 2DMs. b) Nucleation of triangular, epitaxial Au nanoislands on graphene. 

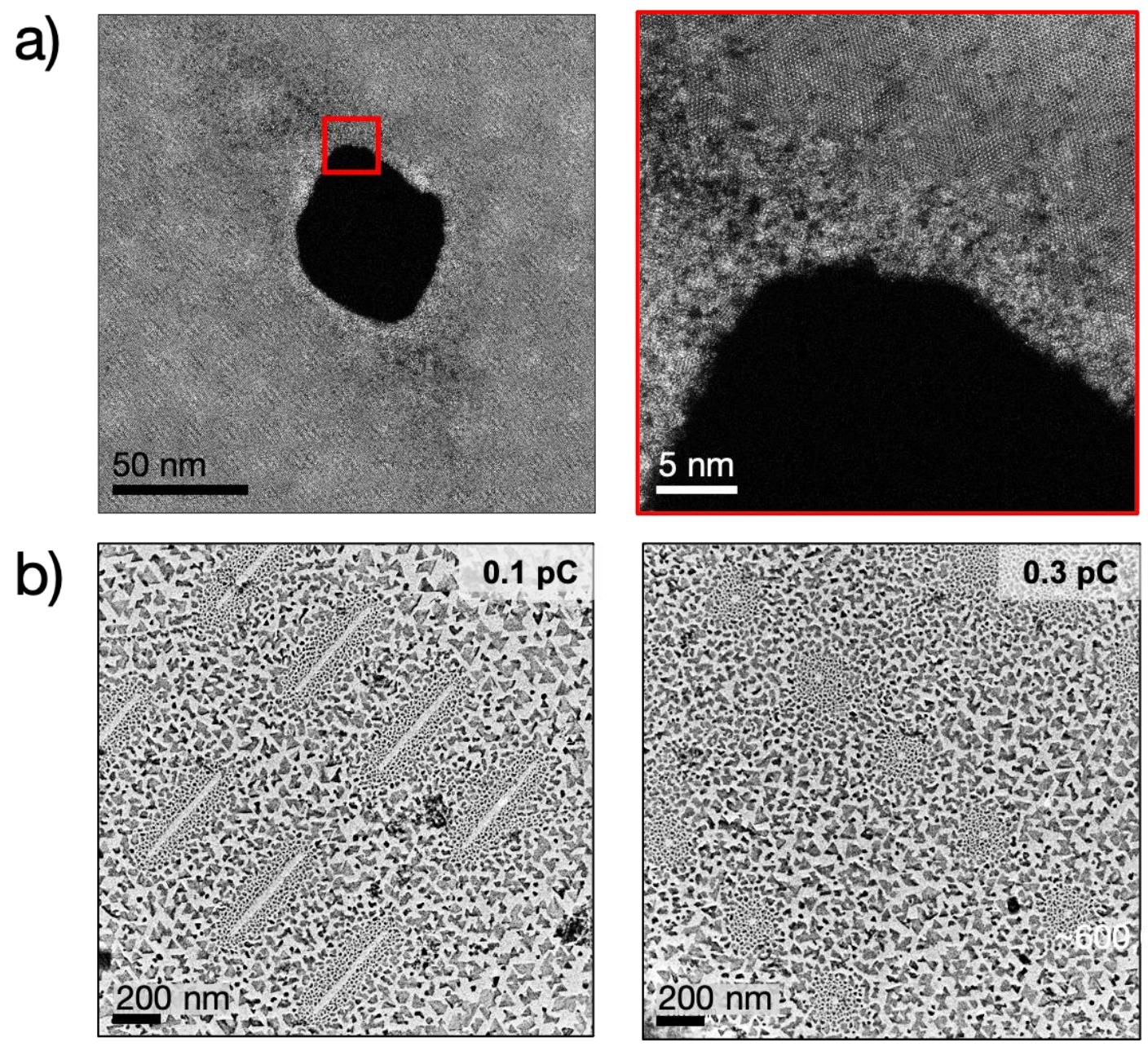

Figure 2. Figure 2: STEM characterization of defects and deposition on defective 2DMs. a) Defect in monolayer MoS2 irradiated with $\mathrm{He}+$ in the HIM showing $50 \mathrm{~nm}$ hole created by $\sim 600,000 \mathrm{He}+$ ions per spot irradiation. Right panel is a high magnification view of the amorphized region around a hole. b) Nucleation of Au islands on irradiated graphene flakes patterned with lines (left) and points (right), where Au appears as dark areas. Inset shows dose used.

\section{References}

[1] J. Bai, et al., Nat. Nanotechnol. 5, 190-194 (2010).

[2] X. Liu, et al., Nat. Commun. 4, (2013).

[3] G. Ye, et al., Nano Lett. 16, 1097-1103 (2016).

[4] S. Malola, et al., Appl. Phys. Lett. 94, (2009).

[5] A. V. Krasheninnikov, et al., Phys. Rev. Lett. (2009).

[6] T. B. Rawal, et al., J. Phys. Chem. C (2017).

[7] W. Ju, et al., Phys. Chem. Chem. Phys. (2017).

[8] J. Zhao, et al., Science 343, 1228-1232 (2014).

[9] S. Antikainen, et al., Comput. Mater. Sci. 131, 120-125 (2017).

[10] X. Li, et al., Chem. Soc. Rev. (2016).

[11] M. Nguyen, et al., Nanoscale 10, 2267-2274 (2018).

[12] T. T. Tran, et al., Nano Letters 17, 2634-2639 (2017).

[13] J. P. Thiruraman, J. P., et al., Adv. Funct. Mater. 29, 1904668 (2019).

[14] D. S. Fox, et al., Nano Lett. 15, 5307-5313 (2015). 
[15] Y. Deng, et al., Nanotechnology 31, (2020).

[16] I. Shorubalko, et al., Beilstein J. Nanotechnol. (2017).

[17] This work made use of facilities and instrumentation supported by NSF through the Massachusetts Institute of Technology Materials Research Science and Engineering Center DMR - 1419807, as well as facilities at MIT.nano. V. Z. acknowledges funding from the Microscopy Society of America Undergraduate Research Scholarship. 